\title{
Effects of Contrast Agent Concentration on the Signal Intensity and Turbo Factor of TSE and Slice-selective IR in T1-weighted Contrast Imaging
}

\author{
Yong Soo Han ${ }^{1}$, Soo Chul Lee ${ }^{1}$, Dong Yong Lee ${ }^{1}$, Jiwon Choi ${ }^{2}$, Jong Woong Lee ${ }^{3}$, and Dae Cheol Kweon ${ }^{4 *}$ \\ ${ }^{1}$ Department of Radiology, Dongguk University Ilsan Medical Center, Goyang 10326, Republic of Korea \\ ${ }^{2}$ Department of Radiological Science, Jeonju University, Jeonju 55069, Republic of Korea \\ ${ }^{3}$ Department of Radiology, Kyung Hee University Hospital at Gang-dong, Seoul 05278, Republic of Korea \\ ${ }^{4}$ Department of Radiologic Science, Shinhan University, Uijeongbu 11644, Republic of Korea
}

(Received 3 September 2015, Received in final form 5 January 2016, Accepted 5 January 2016)

\begin{abstract}
The present study analyzes T1 TSE and T1 slice sel. IR (dark_fluid) signal strength according to the degree of gadolinium contrast agent dilution and analyzes the turbo factors with regard to changes in the maximum and overall signal strength to study correlations between changes and signal-to-noise ratios (SNRs) and compare peak-to-peak SNR (PSNR) enhancement in order to improve the quality of T1-weighted images. Enhancement TR (600 msec) evaluated to determine the T1 TSE turbo factor and obtain the maximum signal strength, T1WI were used sequentially to experiment with turbo factors_1-4. T1 slice sel. IR (dark-fluid) was used to sequentially test turbo factors_2-5 but not turbo factor_1 at a TR $(1500 \mathrm{msec})$ and compare data at an increase in T1 of 900 msec. The T1 TSE was reduced according to the contrast agent concentration. Phantom signal strength increased, whereas turbo factors_1-4 exhibited maximum signal strength at a concentration of 3 mmol, followed by a gradual decrease. In the turbo factors_2-5, the signal strength increased sharply to maximum signal strength at $0.7 \mathrm{mmol}$, followed by a reduction. T1 TSE had a greater maximum signal strength than did T1 slice sel. IR (dark_fluid). A comparison of SNR found that T1 TSE imaging was superior (33.3 dB) in turbo factor_1 and T1 slice sel. IR (dark_fluid) was highest (33.9 dB) at turbo factor_5. A PSNR comparison analysis was not sufficient to distinguish between the images obtained with both techniques at 30 dB or higher under all experimental conditions.
\end{abstract}

Keywords : contrast agent, gadolinium, PSNR, SNR, turbo factor

\section{Introduction}

Magnetic resonance imaging (MRI) has a unique advantage over other imaging methods, as the image contrast is so high that it provides excellent contrasts between soft tissues. This modality also incorporates a variety of conditions under the same organizational process to demonstrate various contrasts in signal intensity between various types of tissue. Brain studies of structural differences associated with image signals, such as neurological proteins (white matter) and gray matter (gray matter), continue to further significant technical developments. Changes in the shading of cerebral microvascular disorders (small vessel disease) might be caused by brain lesions. Substantial brain infarct damage occurs in the

(C)The Korean Magnetics Society. All rights reserved.

*Corresponding author: Tel: +82-31-870-3416

Fax: +82-31-870-3419, e-mail: dckweon@shinhan.ac.kr brain parenchyma, and currently the brain disease site has been proposed as a major risk factor for depression, cognitive depression, stroke, and vascular dementia [1-3]. Brain MRI scans during T1 SE relaxation time do not provide good contrast resolution between the white and gray matter because the inversion recovery (IR) technique, which uses the slice-selective (slice sel.) inversion pulse (dark_fluid imaging) used to increase contrast is considered to be helpful for diagnosing lesions (Fig. 1). Because of this characteristic, initial MRI scans are non-invasive, involve no harmful X-rays, and do not require the use of MR contrast agent; although MR contrast agents affect a limited area, they are widely used to detect tissue characteristics and disease lesions.

The present study analyzes T1 TSE and T1 slice sel. IR (dark_fluid) signal strength according to the degree of gadolinium contrast agent dilution and analyzes the turbo factors with regard to changes in the maximum and overall signal strength to study correlations between changes 


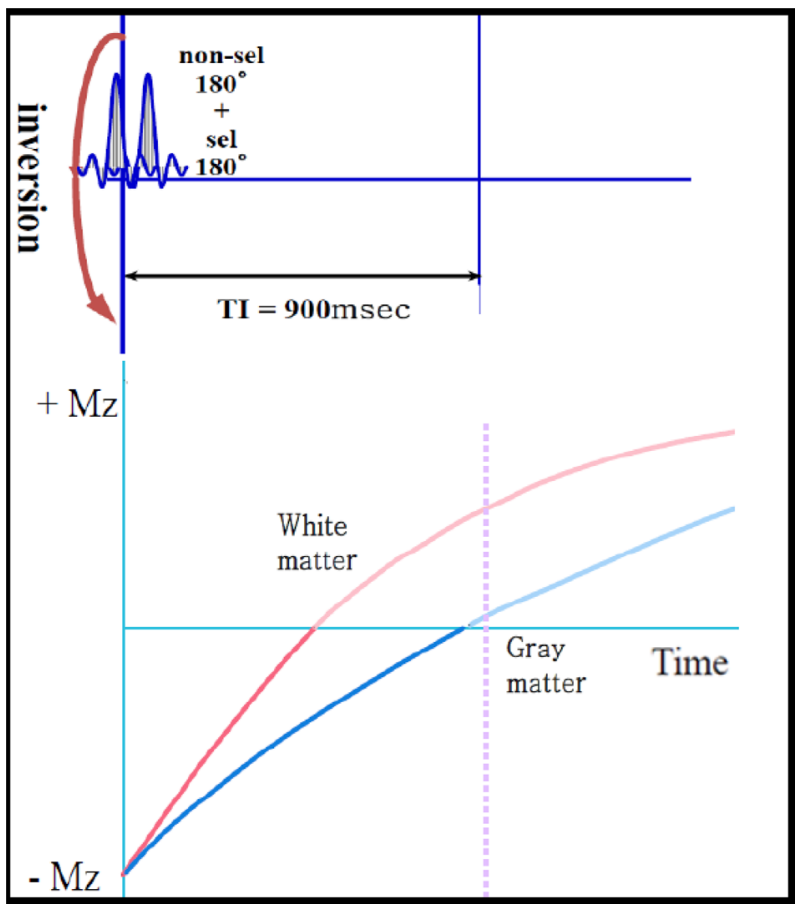

Fig. 1. (Color online) Represent image of inversion recovery.

and signal-to-noise ratios (SNRs) and compare peak-topeak SNR (PSNR) enhancement in order to improve the quality of T1-weighted images.

\section{Theory}

\subsection{MRI}

An MRI radiofrequency pulse excites hydrogen nuclei within the body to a higher energy level, thus causing resonance. After applying this pulse, the protons relaxed to their initial state (i.e., low energy level). In this case, electrical signals are generated via mathematical transformation and then collected with the magnetic resonance signals generated via Fourier transform to obtain MRI and a magnetic resonance spectrum.

\subsection{Contrast agent}

This material is used to highlight tissues and blood vessels and exhibits a high level of contrast in the body during MRI. The MR contrast agent affects the image signal strength both by influencing the magnetic relaxivity of hydrogen and through its distribution [10]. In other words, in the body, contrast agent affects the water molecule relaxation time in both normal and abnormal tissues; by detecting differences in the relaxation time following exposure to a strong external magnetic field and highfrequency energy, contrast between anatomic or functional areas can be maximized $[4,5]$. Upon reaching the bio- logical tissue after injection, the contrast material can indicate changes in the tissue, such as proton density and $\mathrm{T} 1$ and T2 relaxation times, which appear as signals in the image. Accumulation of contrast agent to yield bright signal enhancement is called positive contrast enhancement, whereas accumulation to yield dark enhancement is referred to as negative contrast enhancement. Therefore, in general, an increase in the proton density and a reduction in T1 and T2 appear to promote an increase in signal; in contrast, reductions in proton density and $\mathrm{T} 2$ signal intensity and an increase in $\mathrm{T} 1$ appear to promote a decrease in signal. T1 relaxation effects yield a T1 shortening effect on high-contrast images because of a high concentration of gadolinium per unit volume of [6]. Chelated gadolinium agents act in the extracellular space. These agents distribute through the vascular space and diffuse into interstitial spaces (except the central nervous system and testes) via capillary walls.

\subsection{Turbo spin echo}

TSE provides a similar quality level as conventional spin echo imaging without being influenced by nonuniformity of the main magnetic field; this is achieved by applying the principles of Mansfield echo planar imaging. The study period is longer, as high-speed real-time imaging from the very beginning to the multiple RF echo time is 8-16 times longer than that of the reduced imaging method [15]. The disadvantages of TSE are the creation of 1-shot images of $1 \mathrm{TE}$ because multiple RF echo signals are used to combine different images into a single image [16].

\subsection{Inversion recovery}

IR is a method in which $180^{\circ}$ radiofrequency pulses are applied before the spin echo (SE) provides a $90^{\circ}$ radiofrequency pulse. The use of a $180^{\circ}$ pulse to deliver a high-frequency pulse to each tissue with a known value of zero (null point) and control the reverse T1-recovery time (inversion time, TI) curve can suppress the signal from each tissue [17-19]. Short inversion time IR (short tau inversion recovery, STIR) is a signal of fat tissue suppression, and general increases in the values of $\mathrm{T} 1$ and $\mathrm{T} 2$ lesions are indicated by high signal intensity [17, 20, 21]. A long inversion time inversion recovery IR (fluidattenuated inversion recovery, FLAIR) is a signal of cerebrospinal fluid (CSF) suppression and increases the detection rate of surrounding periventricular lesions or CSF. Inversion time IR may help to diagnose diseases via increased contrast by suppressing the signal of the tissue surrounding the expected lesions. 


\subsection{SNR and PSNR}

The SNR factor, according to changes in TSE with slice sel. IR (dark-fluid), refers to the relative ratio of the signal and noise and indicates image contrast. A comparison of SNR at the signal intensity peak value is indicated below (1).

$$
\begin{aligned}
& S N R[d B]=10 \cdot \log _{10}\left(\frac{P_{\text {signal }}}{P_{\text {noise }}}\right) \\
& P=\frac{1}{N} \sum_{i=0}^{N-1} x^{2}[i]
\end{aligned}
$$

The following metric, which is used often in practice, is called PSNR.

$$
P S N R=10 \cdot \log _{10} \frac{\operatorname{MaxErr}^{2} \cdot w \cdot h}{\sum_{i=0 j=0}^{w \cdot h}\left(x_{i j}-y_{i j}\right)^{2}}
$$

\section{Materials and Methods}

\subsection{Phantom Production}

Gadolinium contrast agent (Gadovist, Bayer Schering, Berlin, Germany) was diluted in normal saline to a concentration of $1000 \mathrm{mmol}$ and used to prepare a self-built phantom that demonstrates changes in signal intensity according to the contrast medium concentration. A 35phantom model was constructed using glass cylinders (height: $11.5 \mathrm{~cm}$, diameter: $2.5 \mathrm{~cm}$ ), and the gadolinium contrast solution was diluted in normal saline at ratios yielding the following concentrations: 400, 300, 200, 100, $90,80,70,60,50,40,30,20,10,9,8,7,6,5,4,3,2,1$, $0.9,0.8,0.7,0.6,0.5,0.4,0.3,0.2,0.1,0.05,0.025$, and $0.0125 \mathrm{mmol}$. The contrast agent dilutions were arranged in the phantom model from high to low concentration (Fig. 2). The experiment was run a total of 5 times to measure the signal strengths of the gadolinium contrast dilutions.

\subsection{Phantom image acquisition methods}

The image acquisition devices included a 3.0-T superconducting MRI (Achieva 3.0T, Siemens Skyra, Germany) (Fig. 2) with a 20-channel head-and-neck coil, and T1 TSE and slice-sel. IR imaging were compared to determine changes in the signal strength during T1 dark_fluid imaging with a turbo factor. At different gadolinium contrast agent concentrations (starting at $1000 \mathrm{mmol}$ ), signal strength data were analyzed and compared in a crossexperiment involving the gadolinium phantom test.

The following image capture conditions were used: for TSE, a field of view (FOV) of $220 \times 220 \mathrm{~mm}$, slice thickness of $5 \mathrm{~mm}, 7$ slices, repetition time (TR) of $600 \mathrm{msec}$,

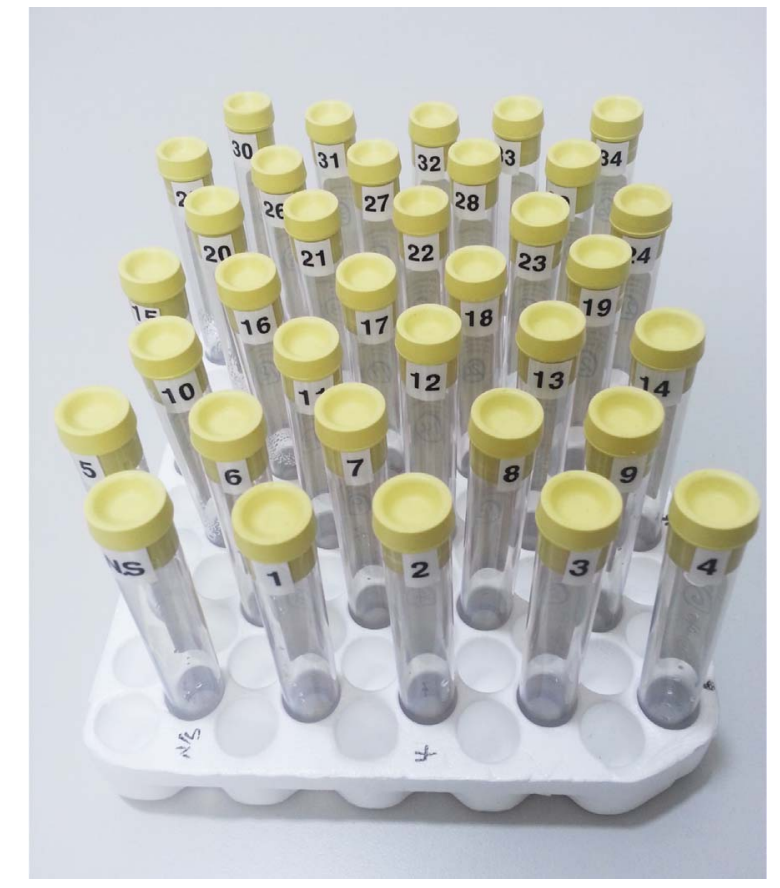

Fig. 2. (Color online) Self-construct phantom to evaluate molar concentrations.

echo time (TE) of $6.4 \mathrm{msec}$, number of excitations (NEX) of 1 , flip angle of $90^{\circ}$, and a matrix of $128 \times 128$; for slice-sel. IR (dark fluid), a FOV of $220 \times 220 \mathrm{~mm}$, slice thickness of $5 \mathrm{~mm}, 7$ slices, TR of $1500 \mathrm{msec}$, TE of 7.5 msec, NEX of 1 , flip angle of $90^{\circ}$, and T1 matrix (slice sel. IR) with a 901-msec condition. Turbo factors_1, 2, 3, and 4 were used for TSE, and turbo factors_2, 3, 4, and 5 were used for slice-sel. IR (dark_fluid) to obtain images of these values. Slice-sel. IR (dark fluid) with an increase in TR of $1500 \mathrm{msec}$ over the TI and turbo factor_1 was excluded from the experiment (Table 1).

\subsection{Comparative analysis}

TSE (Fig. 3) and slice sel. IR (Fig. 4) images were

Table 1. Scan parameters.

\begin{tabular}{ccc}
\hline \hline Parameter & T1 TSE & $\begin{array}{c}\text { T1 slice sel. IR } \\
\text { (dark_fluid) }\end{array}$ \\
\hline FOV $(\mathrm{mm})$ & $220 \times 220$ & $220 \times 220$ \\
Slice thickness $(\mathrm{mm})$ & 5 & 5 \\
Slice $(\mathrm{mm})$ & 7 & 7 \\
TR $(\mathrm{msec})$ & 600 & 1500 \\
TE $(\mathrm{msec})$ & 6.4 & 7.5 \\
NEX & 1 & 1 \\
Flip angle (degrees) & 90 & 90 \\
Matrix & $128 \times 128$ & $128 \times 128$ \\
\hline
\end{tabular}




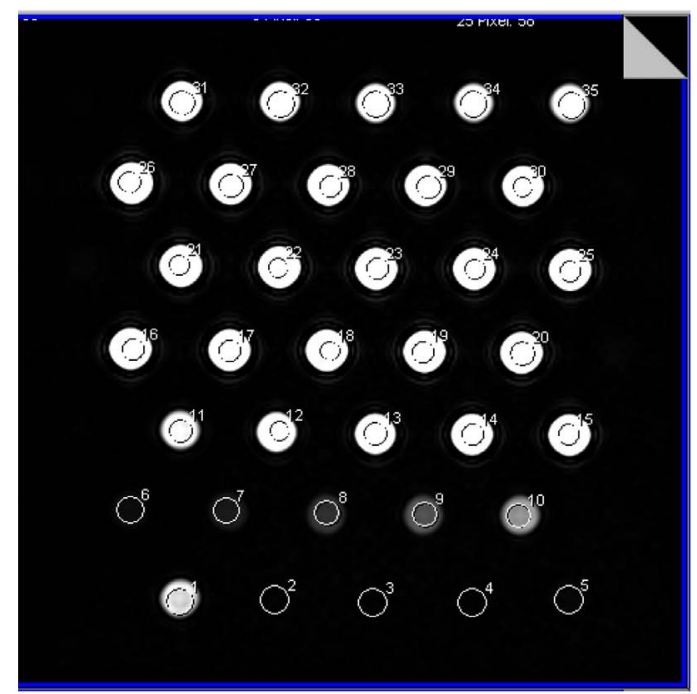

(a)

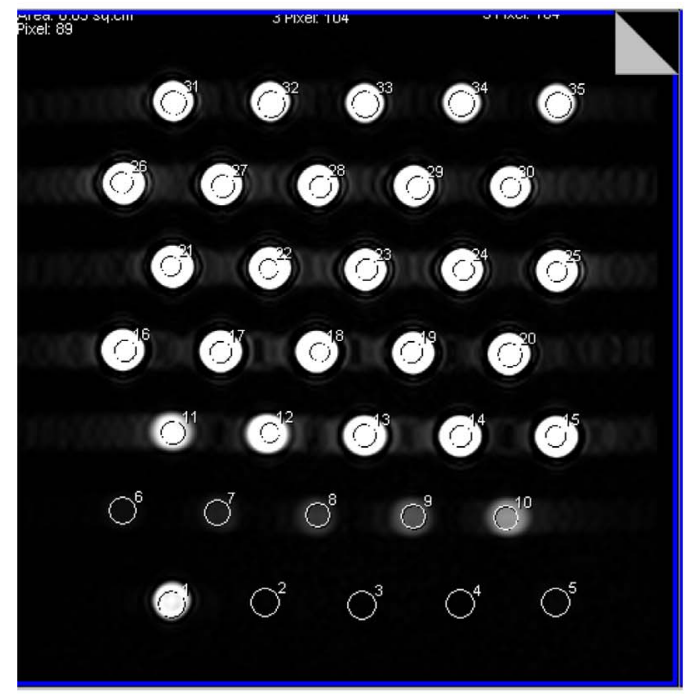

(c)

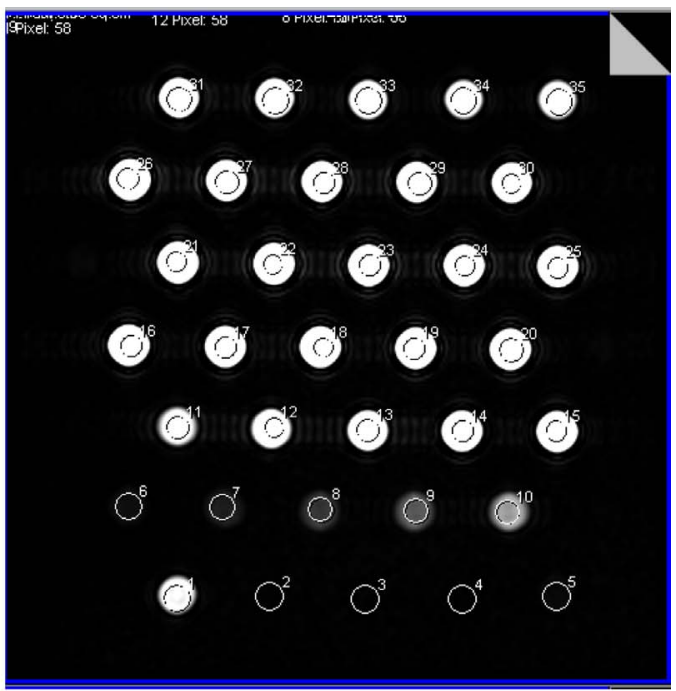

(b)

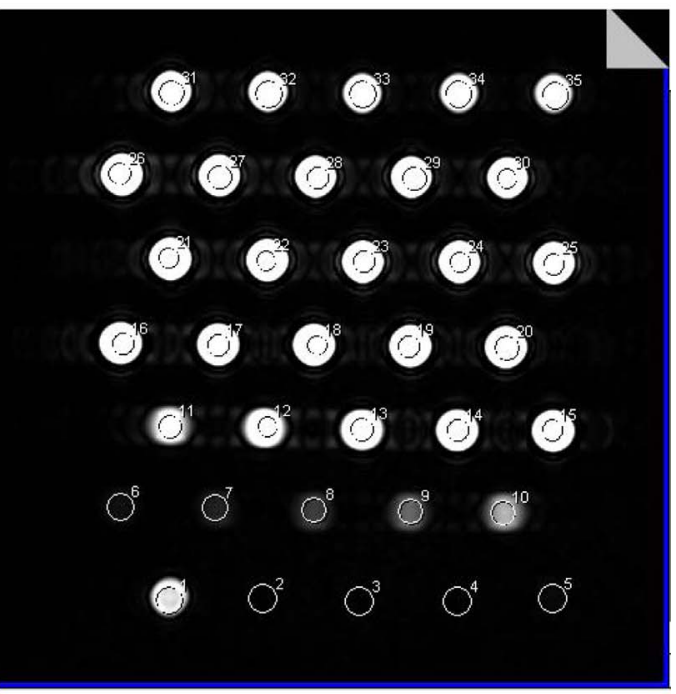

(d)

Fig. 3. (Color online) Measurement of turbo spin-echo (TSE) signal intensity. (a) T1 TSE (turbo-1), (b) T1 TSE (turbo-2), (c) T1 TSE (turbo-3), (d) T1 TSE (turbo-4).

analyzed using a Syngovia (Siemens AG, Erlangen, Germany) to measure the signal intensities in the phantom images at diluted contrast agent concentrations ranging from 0.0125$400 \mathrm{mmol}$ by setting regions of interest (ROIs).

An evaluation of image noise, or PSNR, was used to evaluate losses in image quality on T1 TSE and T1 slicesel. IR (dark_fluid) images; accordingly, small losses of information received high values $(30<\mathrm{PSNR} \leq 50)$. The MSU Video Quality Measurement Tool (Graphics \& Media Lab Video Group) was used to compare the PSNRs.

\section{Results}

The signal strength of each contrast ratio dilution was measured against each of the others in glass cylinders to determine contrast phantom signal strength properties in a phantom test using T1 TSE and T1 slice sel. IR (dark_fluid); the turbo factor number and signal intensity were analyzed in accordance with the contrast agent dilution. As a result, when T1 TSE images of 2 gadolinium contrast agent dilutions were compared at turbo factor_1, turbo factor_2, turbo factor_3, and turbo factor_4, the maximum signal strength of 3366.2 was observed at $3 \mathrm{mmol}$ and turbo factor 3 (Fig. 5). In addition, the signal strength decreased relatively gradually beginning at a gadolinium contrast agent dilution of $0.1 \%$ while maintaining a maximum signal strength in the overall dilution range of $0.3-0.07 \%$ (Table 2). 


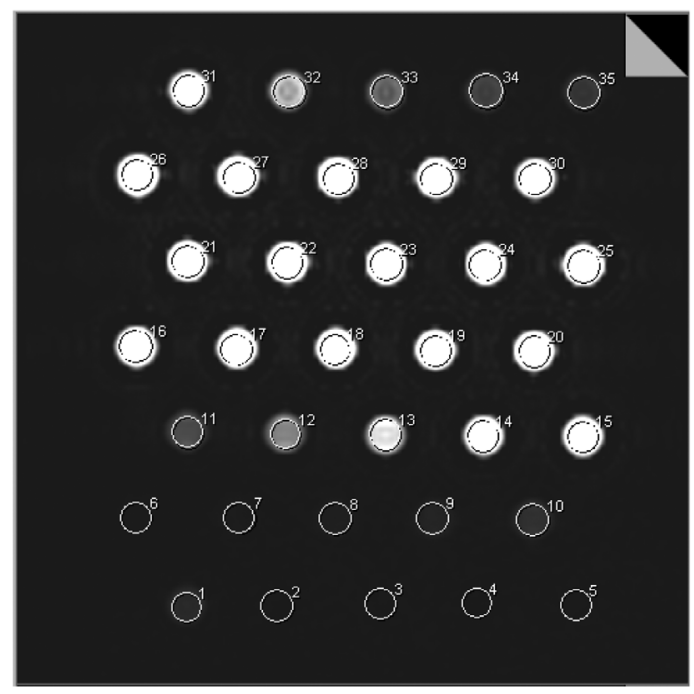

(a)

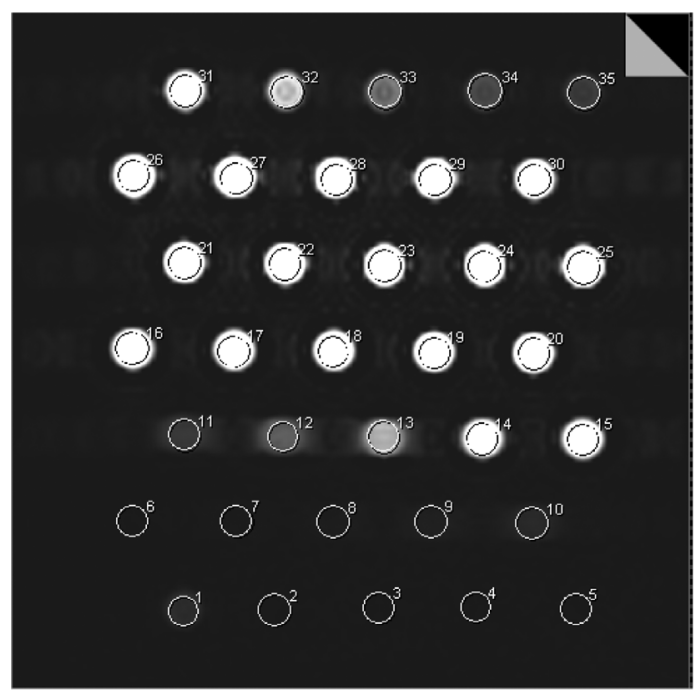

(c)

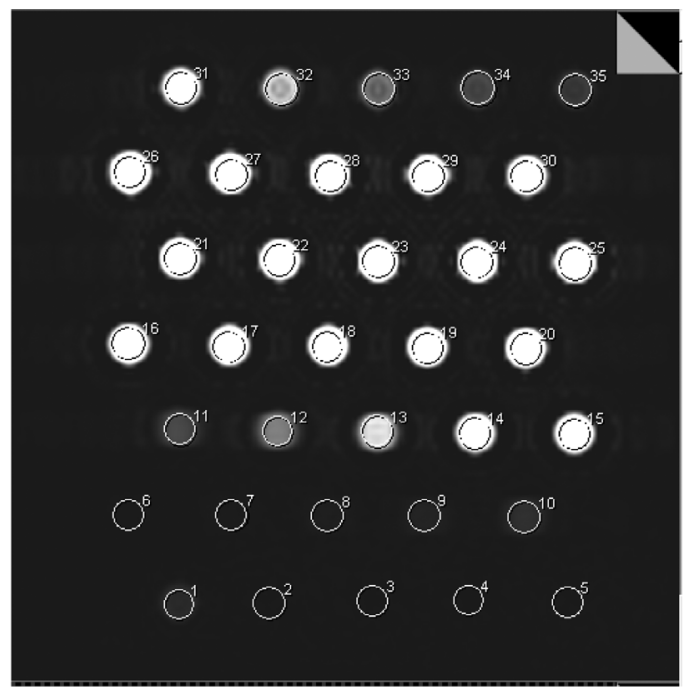

(b)

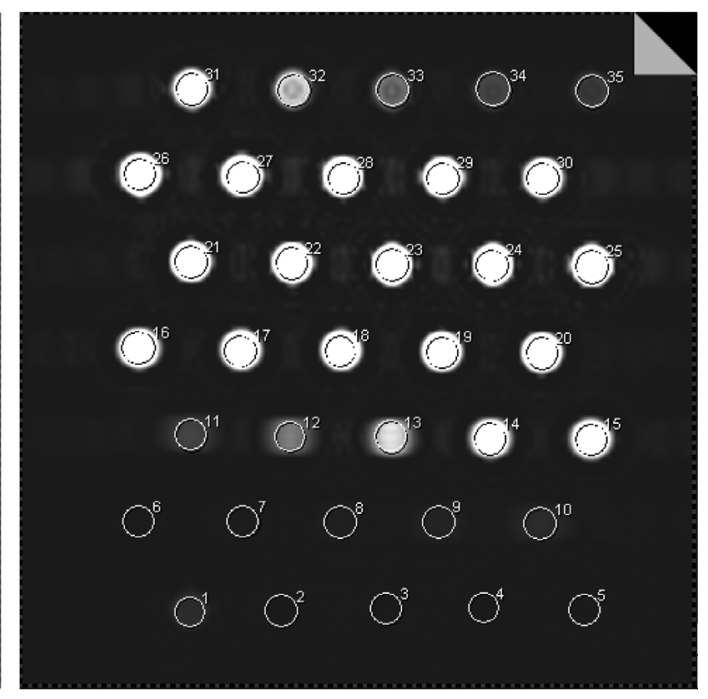

(d)

Fig. 4. Measurement of $\mathrm{T} 1$ slice-selected inversion recovery (dark_fluid) signal intensity. (a) T1 tirm dark fluid (factor-2), (b) T1 tirm dark fluid (factor-3), (c) T1 tirm dark fluid (factor-4), (d) T1 tirm dark fluid (factor-5).

Next, the maximum signal strengths from both imaging techniques were compared to determine the obtained values; T1 slice sel. IR (dark fluid) (glass cylinder 25, concentration of $0.7 \mathrm{mmol}$, turbo factor_4) exhibited a maximum signal strength at a more dilute concentration than that achieved with T1 TSE ( $3 \mathrm{mmol}$, glass cylinder 20, turbo factor_3) (Fig. 6). A maximum signal strength comparison of the 2 conditions revealed that the absolute value achieved with T1 TSE, 3366.2, was higher than that achieved with T1 slice sel. IR (dark fluid), 3246.9 (Fig. 7, Table 3).

An SNR comparison of T1 TSE results yielded the following values: turbo factor_1, $33.308 \mathrm{~dB}$; turbo factor_2, $32.703 \mathrm{~dB}$; turbo factor_3, $33.230 \mathrm{~dB}$; and turbo factor 4, $33.208 \mathrm{~dB}$ (Table 4). A similar comparison of T1 slice sel. IR (dark_fluid) results yielded the following SNR values: turbo factor $2,32.831 \mathrm{~dB}$; turbo factor 3 , $33.153 \mathrm{~dB}$; turbo factor $4,31.892 \mathrm{~dB}$; and turbo factor_5, $33.900 \mathrm{~dB}$ (Table 5). As a result, T1 TSE achieved best results at turbo factor_1, with an increase in value to $33.308 \mathrm{~dB}$, then exhibited a slight decrease in turbo factor_2 and another increase in turbo factor_3. T1 slice sel. IR (dark fluid) gradually increased from the turbo factor_2, with a slight decrease in turbo factor_4 (31.892 $\mathrm{dB}$ ) before reaching a peak of $33.900 \mathrm{~dB}$ at turbo factor_5.

A PSNR comparison of T1 TSE results, based on turbo factor_1, yielded the following values: turbo factor_1, 50 


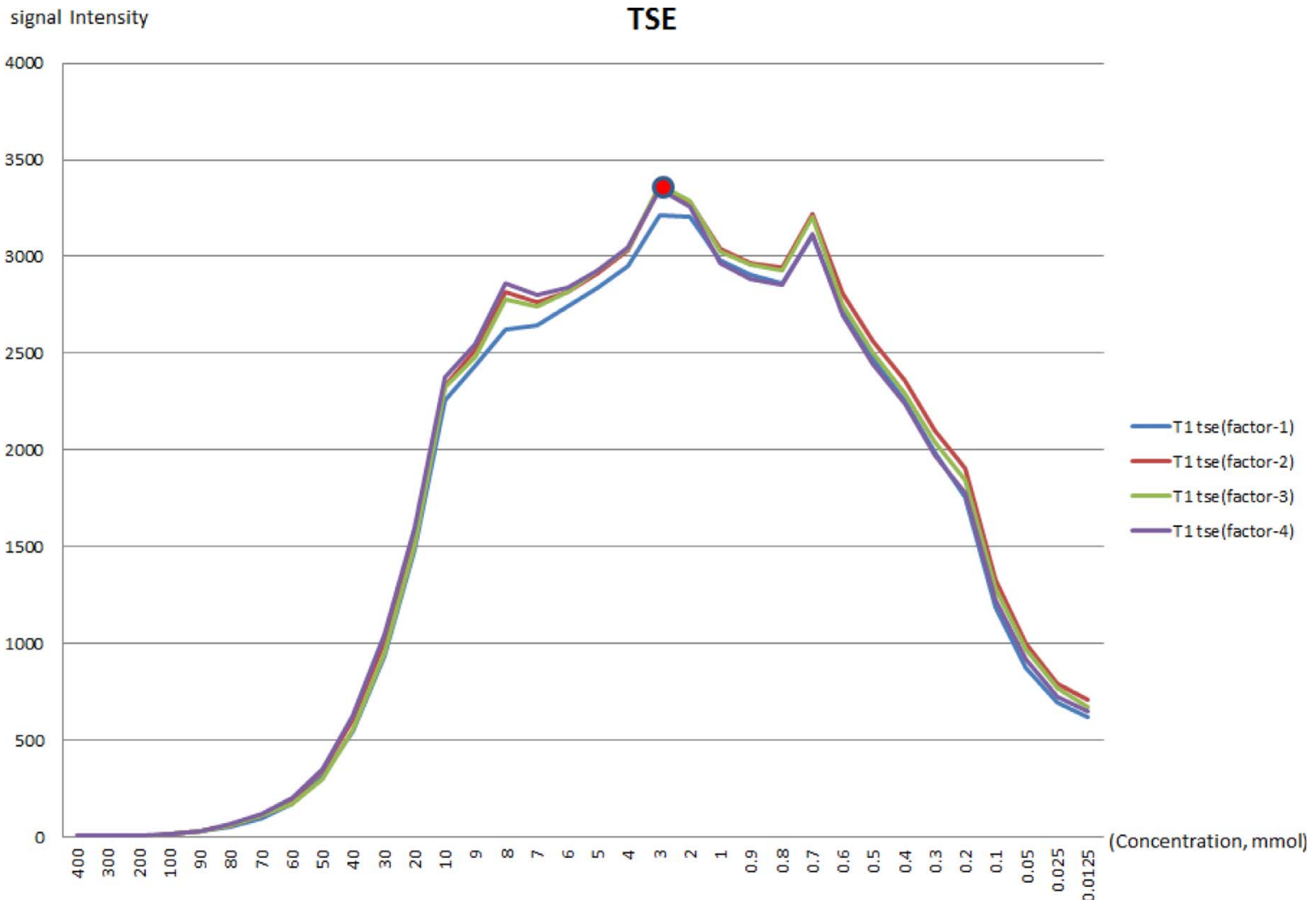

Fig. 5. (Color online) Change of T1 turbo spin-echo (TSE) of response to contrast concentration.

$\mathrm{dB}$; turbo factor_2, $39.3276 \mathrm{~dB}$; turbo factor_3, 40.2530 $\mathrm{dB}$; and turbo factor_4, $39.8237 \mathrm{~dB}$ (Table 4). A similar comparison of T1 slice sel. IR (dark_fluid) PSNR values, based on turbo factor_2, yielded the following: turbo factor_2, $50 \mathrm{~dB}$; turbo factor_3, $49.0101 \mathrm{~dB}$; turbo factor_4, $48.7432 \mathrm{~dB}$; and turbo factor_5, $45.3303 \mathrm{~dB}$ (Table 5). As a result, T1 TSE had the highest PSNR, 50 $\mathrm{dB}$, at turbo factor_1 and the lowest PSNR, $39.3276 \mathrm{~dB}$, at turbo factor_2. T1 slice sel. IR (dark_fluid) had the highest PSNR, $50 \mathrm{~dB}$, at turbo factor_2 and the lowest value, $45.3303 \mathrm{~dB}$, at turbo factor_5.

\section{Discussion}

Contrast-enhanced T1-weighted images are important primarily for the diagnosis of diseases such as brain inflammation, abscesses, or tumors. Lesions that affect the blood-brain barrier can destroy contrast enhancement in the brain parenchyma, resulting in gadolinium contrast agent movement. For the same reason, acute cerebral infarction appears to enhance brain parenchyma contrast in substantial brain imaging scans. However, contrastenhanced blood vessel blockages in areas of brain infarction reduce the intravascular blood volume, and because of this automatic blood vascular adjustment mechanism, contrast spreads slowly through the blood flow within a blood vessel; following the accumulation of gadolinium contrast agents in blood vessels and early signs of cerebral infarction, the appearance of signal strength and infarction symptoms are often seen in examinations performed within 24 hours but disappear within a few days [14]. Therefore, the actual size of the enhanced signal strength area may be very important.

We evaluated and confirmed the effect of a contrast agent concentration via dilution on signal intensity through a real contrast agent gadolinium phantom test, a sustainability evaluation of the contrast effect by comparing signal strengths at the maximum signal strength, and most significantly confirmed a 0.0125 -mmol dilution throughout the imaging experiment by comparison. The factor that increases contrast-to-noise ratio (CNR) enhancement and image acquisition when SNR is determined is as follows:

First, the main magnetic field is larger; SNR and CNR indicated this increase in contrast effect in the brain (brain tumor) and multiple sclerosis (MS) $[15,16]$.

Second, at a high gadolinium contrast agent concentration $(1 \mathrm{mmol} / \mathrm{ml}), \mathrm{SNR}$ and $\mathrm{CNR}$ can be obtained for 
Table 2. Signal intensity changes associated with contrast concentrations in T1 TSE.

\begin{tabular}{|c|c|c|c|c|c|c|c|c|}
\hline \multirow{2}{*}{$\begin{array}{c}\text { Sequence } \\
\text { Concentration } \\
(\mathrm{mmol})\end{array}$} & \multicolumn{2}{|c|}{ T1 TSE (factor-1) } & \multicolumn{2}{|c|}{ T1 TSE (factor-2) } & \multicolumn{2}{|c|}{ T1 TSE (factor-3) } & \multicolumn{2}{|c|}{ T1 TSE (factor-4) } \\
\hline & Mean & SD & Mean & SD & Mean & SD & Mean & SD \\
\hline 400 & 5 & 1.7 & 5 & 2.3 & 6.2 & 2.2 & 4.4 & 2 \\
\hline 300 & 5 & 2.1 & 5.4 & 2.4 & 6.1 & 2.1 & 6.7 & 2.4 \\
\hline 200 & 4.3 & 1.8 & 4.1 & 1.5 & 4.1 & 1.6 & 4.5 & 1.8 \\
\hline 100 & 15.1 & 2.4 & 18.1 & 2.4 & 14.5 & 2.6 & 17.2 & 3.4 \\
\hline 90 & 26.8 & 1.9 & 32.2 & 2.7 & 28.8 & 2 & 32 & 4.1 \\
\hline 80 & 55.5 & 3.2 & 63.6 & 4.8 & 61.8 & 4.2 & 64.3 & 9.7 \\
\hline 70 & 101.2 & 3.5 & 116.2 & 6.1 & 110.4 & 5.4 & 120.1 & 10.8 \\
\hline 60 & 173.4 & 5.3 & 194.5 & 9.4 & 174.9 & 7.2 & 204.2 & 19.7 \\
\hline 50 & 315.3 & 12.4 & 342.8 & 19.4 & 300.8 & 14.9 & 348.4 & 45.1 \\
\hline 40 & 548.3 & 20.8 & 609.4 & 36.3 & 552.6 & 18.1 & 630.1 & 48.6 \\
\hline 30 & 934.7 & 38.4 & 1007.8 & 48.2 & 951 & 15.7 & 1047.4 & 53 \\
\hline 20 & 1485.7 & 82.7 & 1564.2 & 87.2 & 1509.1 & 47.1 & 1598.2 & 69.2 \\
\hline 10 & 2257.7 & 99.9 & 2329.9 & 105.1 & 2322.5 & 81.6 & 2377.6 & 81.1 \\
\hline 9 & 2434.3 & 126 & 2516.3 & 125.4 & 2482.9 & 103.9 & 2544.7 & 100.9 \\
\hline 8 & 2623.2 & 119.3 & 2820.2 & 138.5 & 2780.6 & 128.4 & 2864.5 & 113.2 \\
\hline 7 & 2647.2 & 103 & 2763.3 & 112.1 & 2744.7 & 93.4 & 2799 & 92.6 \\
\hline 6 & 2739.2 & 128.6 & 2819.9 & 131.4 & 2820.4 & 108.8 & 2842.4 & 111.2 \\
\hline 5 & 2842.3 & 152.1 & 2914.9 & 157.5 & 2932.4 & 143.8 & 2928.7 & 143.8 \\
\hline 4 & 2950.1 & 153 & 3034.8 & 160.7 & 3040.6 & 147.2 & 3045.9 & 159.1 \\
\hline 3 & 3213.3 & 175.1 & 3354.6 & 186.3 & 3366.2 & 203.9 & 3349.1 & 192.7 \\
\hline 2 & 3205.3 & 154 & 3281.5 & 162.6 & 3289.5 & 171.4 & 3254.1 & 176.1 \\
\hline 1 & 2978.4 & 120.4 & 3040 & 127.2 & 3024.4 & 134.1 & 2965.3 & 147.2 \\
\hline 0.9 & 2903.6 & 113.2 & 2967.9 & 124.3 & 2960.7 & 131 & 2882.1 & 146.4 \\
\hline 0.8 & 2861.9 & 100.8 & 2947.3 & 107.3 & 2929.6 & 117 & 2853.2 & 127.9 \\
\hline 0.7 & 3106.3 & 213.5 & 3217 & 224.9 & 3203.2 & 278 & 3113.1 & 254 \\
\hline 0.6 & 2720.2 & 125.1 & 2810.2 & 134.4 & 2751.5 & 146.5 & 2697.5 & 153.2 \\
\hline 0.5 & 2474.3 & 110.5 & 2563.8 & 119.3 & 2500.6 & 115.9 & 2445.7 & 134.3 \\
\hline 0.4 & 2257.8 & 106.1 & 2359.5 & 113.3 & 2295.4 & 117.6 & 2239.3 & 136.2 \\
\hline 0.3 & 1989.4 & 114.6 & 2098.2 & 125.6 & 2039.6 & 143.7 & 1974 & 139.1 \\
\hline 0.2 & 1753.9 & 119.6 & 1905.3 & 130.3 & 1848.8 & 147.3 & 1779.9 & 140.7 \\
\hline 0.1 & 1186.6 & 81.5 & 1332.5 & 95.4 & 1282.7 & 100.5 & 1224.5 & 100.1 \\
\hline 0.05 & 873.1 & 61.6 & 1004.8 & 73.5 & 969.5 & 83.8 & 919.1 & 78.3 \\
\hline 0.025 & 694.2 & 43.2 & 795.3 & 51.3 & 769.8 & 60.7 & 724.2 & 50.6 \\
\hline 0.0125 & 623.8 & 36.2 & 709.2 & 43.3 & 671.9 & 46.7 & 647.4 & 44.1 \\
\hline Normal saline & 474.3 & 25.4 & 553.4 & 31 & 543.2 & 32.9 & 502.4 & 31.9 \\
\hline
\end{tabular}

the increased image value $[17,18]$.

However, these studies reported only differences relative to the main magnetic field and gadolinium concentration, but a difference was still observed. This study evaluated enhancement under the same physical conditions and compared differences in the changes in signal strength and SNR in T1-weighted images obtained with the inspection techniques. Therefore, this study used a 3.0-T high magnetic field device with gadolinium contrast and a T1 relaxation rate; a greater contrast concentration $(1,000$ $\mathrm{mmol} / \mathrm{ml}$ ) was used to minimize the impact on the resting condition.

\section{Conclusions}

Post-contrast T1 TSE and T1 slice sel. IR (dark_fluid) images compared with images obtained at different turbo factors revealed the following maximum signal strengths during the gadolinium contrast agent phantom test: T1 TSE (glass cylinder 20, concentration of $3 \mathrm{mmol}$, turbo factor_3) of 3366.2 and T1 slice sel. IR (dark_fluid) (glass cylinder 25, concentration of $0.7 \mathrm{mmol}$, turbo factor 4) of 3246.9. T1 TSE exhibited greater maximum signal strength than did T1 slice sel. IR (dark_fluid). The experiment further confirmed that after reaching the 


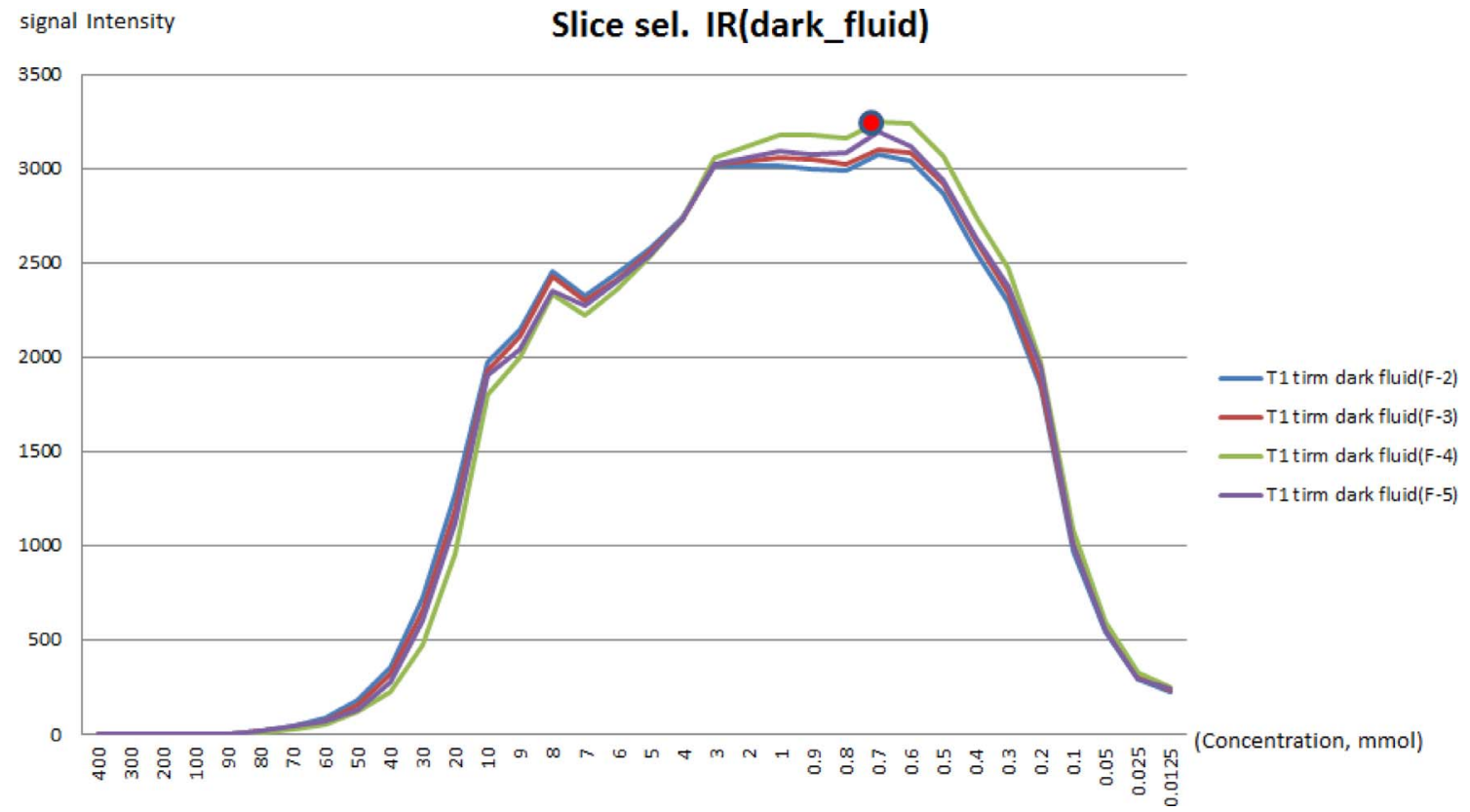

Fig. 6. (Color online) Changes in $\mathrm{T} 1$ slice selected (sel.) inversion recovery (IR) (dark_fluid) in response to contrast concentration.

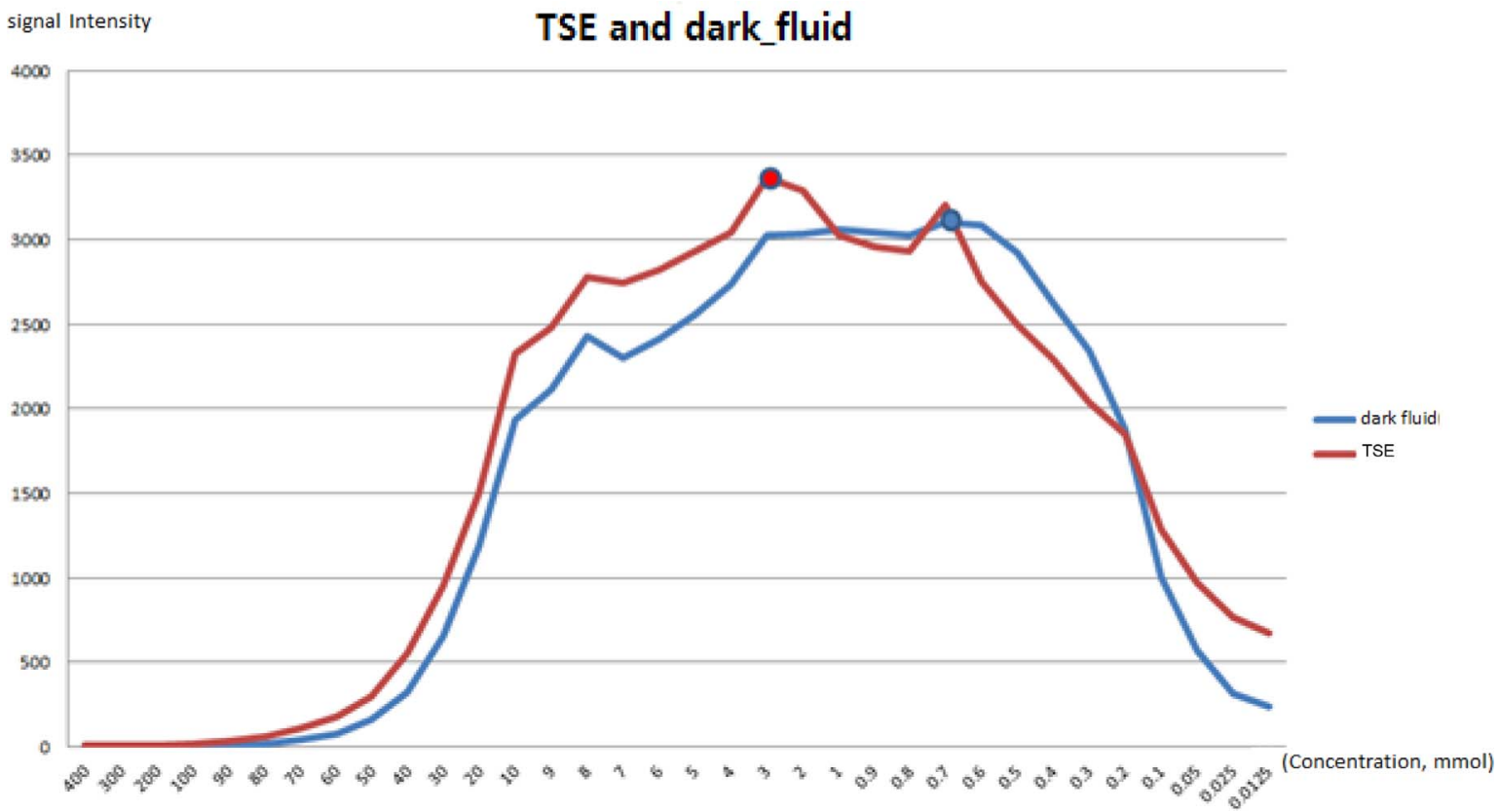

Fig. 7. (Color online) Comparison of T1 turbo spin-echo (TSE) and T1 slice-selected (sel.) inversion recovery (dark fluid) according concentration of contrast.

maximum signal strength, T1 TSE exhibited a gradual decrease that was slower than that observed with T1 slice sel. IR (dark fluid). The greatest signal strengths at a 0.0125 -mmol dilution were 671.9 with T1 TSE and 252.2 with T1 slice sel. IR (dark_fluid). The contrast effect is therefore expected to have a longer duration in T1 TSE. When the SNR results obtained with both techniques were compared, T1 TSE imaging exhibited a slight difference (1\%) and the highest value of $33.308 \mathrm{~dB}$ at turbo factor_1, whereas T1 slice sel. IR (dark_fluid) exhibited the highest value of $33.900 \mathrm{~dB}$ at turbo factor 5 and a difference of $<6 \%$. In a PSNR comparison, T1 TSE exhibited a difference of $>20 \%$ at the standard turbo factor_1, with a value of $50 \mathrm{~dB}$, and $\mathrm{T} 1$ slice sel. IR 
Table 3. Signal intensity change associated with contrast concentration in T1 slice-selected inversion recovery (dark_fluid).

\begin{tabular}{|c|c|c|c|c|c|c|c|c|}
\hline \multirow{2}{*}{$\begin{array}{c}\text { Sequence } \\
\text { Concentration } \\
(\mathrm{mmol})\end{array}$} & \multicolumn{2}{|c|}{ T1 tirm dark fluid (factor-2) } & \multicolumn{2}{|c|}{ T1 tirm dark fluid (factor-3) } & \multicolumn{2}{|c|}{ T1 tirm dark fluid (factor-4) } & \multicolumn{2}{|c|}{ T1 tirm dark fluid (factor-5)) } \\
\hline & Mean & SD & Mean & SD & Mean & SD & Mean & SD \\
\hline 400 & 4.7 & 2 & 5.1 & 1.7 & 6 & 2.2 & 5.2 & 1.8 \\
\hline 300 & 5.8 & 1.8 & 5.5 & 1.9 & 5.4 & 1.7 & 5.9 & 1.8 \\
\hline 200 & 4.1 & 1 & 4.7 & 1.7 & 5.5 & 1.6 & 4.2 & 1.3 \\
\hline 100 & 6.6 & 2 & 5.4 & 2.1 & 5.2 & 2.1 & 5.4 & 2 \\
\hline 90 & 7.8 & 2.3 & 7.1 & 1.8 & 5.2 & 1.4 & 7 & 1.6 \\
\hline 80 & 20.5 & 5.5 & 18.3 & 4 & 13.4 & 3.4 & 19.5 & 5 \\
\hline 70 & 46.5 & 8.6 & 40.4 & 5.9 & 29.2 & 5.5 & 43.4 & 6.3 \\
\hline 60 & 85.9 & 13.9 & 76.6 & 11.7 & 54.4 & 6.7 & 71.9 & 10.7 \\
\hline 50 & 180.5 & 24.9 & 162.5 & 26.4 & 122 & 15.9 & 129.1 & 21.3 \\
\hline 40 & 355.1 & 73.8 & 318.4 & 73.2 & 229.6 & 48.9 & 275.8 & 358.1 \\
\hline 30 & 729.6 & 98.6 & 656.6 & 97.4 & 475.5 & 59.7 & 602.2 & 99.9 \\
\hline 20 & 1276.4 & 118.9 & 1193.5 & 132.9 & 959.1 & 93.5 & 1119.3 & 159.8 \\
\hline 10 & 1976.4 & 392.7 & 1930.6 & 369.9 & 1799.6 & 299.6 & 1908.9 & 374.5 \\
\hline 9 & 2147.8 & 405.7 & 2110.9 & 404.4 & 2003.5 & 358.1 & 2042.7 & 396.4 \\
\hline 8 & 2451.3 & 417.9 & 2427.1 & 416.4 & 2338.3 & 365 & 2354.6 & 414.7 \\
\hline 7 & 2328.8 & 528.6 & 2298.4 & 532.6 & 2221.5 & 488.3 & 2277.5 & 535.2 \\
\hline 6 & 2444.4 & 255 & 2415.6 & 463.1 & 2359.5 & 421.5 & 2407.4 & 400.7 \\
\hline 5 & 2575.8 & 359 & 2914.9 & 369.2 & 2533.1 & 337.7 & 2544.1 & 364.6 \\
\hline 4 & 2742.4 & 251.1 & 2734.7 & 259.8 & 2733.1 & 247.8 & 2726.7 & 271.2 \\
\hline 3 & 3015.7 & 281.4 & 3025.9 & 294.1 & 3062 & 288.2 & 3024.2 & 309.4 \\
\hline 2 & 3016.6 & 376.8 & 3038.6 & 391.5 & 3119.2 & 386.4 & 3053.6 & 394.4 \\
\hline 1 & 3017.2 & 505.8 & 3058.8 & 511.6 & 3178.2 & 528.5 & 3093 & 526.1 \\
\hline 0.9 & 3001.1 & 498.1 & 3046.3 & 502.1 & 3177.1 & 523.5 & 3075.4 & 513.5 \\
\hline 0.8 & 2985.7 & 607.7 & 3024 & 627.4 & 3162.1 & 641.8 & 3079.8 & 648.5 \\
\hline 0.7 & 3070.8 & 862.5 & 3100.3 & 873.1 & 3246.9 & 897.1 & 3191.4 & 900.1 \\
\hline 0.6 & 3043.2 & 334.3 & 3082.5 & 346.5 & 3237.4 & 386.2 & 3119.8 & 360.4 \\
\hline 0.5 & 2870.9 & 274.7 & 2922.7 & 292.8 & 3065.6 & 315 & 2941.5 & 293.4 \\
\hline 0.4 & 2562.1 & 522.9 & 2621 & 544.9 & 2751 & 548.8 & 2635.7 & 556.8 \\
\hline 0.3 & 2295.1 & 385.1 & 2845.6 & 400.7 & 2469.4 & 405.9 & 2375.3 & 413.8 \\
\hline 0.2 & 1845.7 & 382.7 & 1874.5 & 393.5 & 1976.7 & 401.6 & 1947.4 & 411.6 \\
\hline 0.1 & 976.4 & 223.7 & 1006.4 & 229.1 & 1087.5 & 237.9 & 1013.8 & 233.2 \\
\hline 0.05 & 541.8 & 69.2 & 569.2 & 74.7 & 598.5 & 78.5 & 555.4 & 67.8 \\
\hline 0.025 & 293.3 & 63 & 314.6 & 68.8 & 331.6 & 71.9 & 300.4 & 65 \\
\hline 0.0125 & 224.3 & 36.5 & 237.5 & 40.2 & 252.2 & 45 & 240.9 & 41.5 \\
\hline Normal saline & 135.2 & 10.2 & 142.1 & 12.7 & 154.5 & 12.6 & 155.9 & 13.6 \\
\hline
\end{tabular}

Table 4. T1 Turbo spin echo signal-to-noise ratio (SNR) and peak-to-peak SNR (PSNR).

\begin{tabular}{ccccc}
\hline \hline & T1 SE (factor-1) & T1 TSE (factor-2) & T1 TSE (factor-3) & T1 TSE (factor-4) \\
\hline SNR (dB) & 33.308 & 32.703 & 33.230 & 33.208 \\
PSNR (dB) & 50 & 39.3276 & 40.2530 & 39.8237 \\
\hline
\end{tabular}

Table 5. T1 slice-selected inversion recovery (IR) (dark_fluid) of signal-to-noise ratio (SNR) and peak-to-peak SNR (PSNR).

\begin{tabular}{ccccc} 
& T1 tirm dark fluid (factor-2) & T1 tirm dark fluid (factor-3) & T1 tirm dark fluid (factor-4) & T1 tirm dark fluid (factor-5) \\
\hline SNR (dB) & 32.831 & 33.153 & 31.892 & 33.900 \\
PSNR (dB) & 50 & 49.0101 & 48.7432 & 45.3303 \\
\hline
\end{tabular}


(dark_fluid) exhibited a negative difference of $50 \mathrm{~dB}$, less than the standard $10 \%$ of turbo factor_1. However, the data were not sufficient to distinguish visible differences between images of $30 \mathrm{~dB}$ or greater under all experimental conditions.

Clinical trials have not previously conducted an experiment involving a self-constructed phantom containing gadolinium contrast agent dilutions in normal saline, and this study had some limitations. However, the greater value identified in this study is that obtaining the optimal contrast effect would be useful for imaging during postenhancement acquisition using T1 TSE T1-weighted images and T1 slice sel. IR (dark_fluid).

\section{References}

[1] J. Masuda, T. Nabika, and Y. Notsu, Curr. Opin. Neurol. 14, 77 (2001).

[2] W. D. Taylor, M. E. Payne, K. R. Krishnan, H. R. Wagner, J. M. Provenzale, D. C. Steffens, and J. R. MacFall, Biol. Psychiatry 50, 179 (2001).

[3] K. R. Krishnan, Am. Heart J. 140, 70 (2000).

[4] J. H. Choi, S. M. Lim, and Y. Kim, J. Korean Radiol. Soc. 64, 317 (2011).

[5] B. J. Park, M. G. Kim, S. I. Suh, S. J. Hong, K. R. Cho, B. K. Seo, K. Y. Lee, N. J. Lee, and J. H. Kim, J. Korean Med. 44, 317 (2001).

[6] K. W. Choi, S. Y. Son, T. H. Kim, M. S. Han, J. H. Lee, and J. W. Min, J. Korean Radiol. Soc. 14, 1294 (2013).

[7] F. A. Jolesz, Diagn. Imaging 6, 78 (1992).
[8] M. H. Cho, S. Y. Lee, C. W. Mun, H. H. Cho, W. Yi, and W. M. Choi, J. Biomed. Eng. Res. 19, 91 (1998).

[9] S. W. Atlas, R. I. Grossman, D. B. Hackney, H. I. Goldberg, L. T. Bilaniuk, and R. A. Zimmerman, Am. J. Roentgenol. 151, 1515 (1988).

[10] K. M. Jones, R. B. Schwarts, M. T. Mantello, S. S. Ahn, R. Khorasani, S. Mukherji, K. Oshio, and R. V. Mulkern, Am. J. Neuroradiol. 15, 401 (1994).

[11] L. J. Wolnsky, A. Evans, K. Belitsis, P. D. Shaderowfsky, R. Gonzales, J. A. Maldjian, H. J. Lee, and J. Pak, Clin. Imaging 20, 164 (1996).

[12] G. M. Bydder and I. R. Young, J. Comput. Assist. Tomogr. 9, 659 (1985).

[13] R. C. Smith, R. T. Contrast, C. Reinhol, T. McCauley, R. C. Lange, and S. McCarthy, Comput. Assist. Tomogr. 18, 209 (1994).

[14] D. P. Mueller, W. T. Yuh, D. J. Fisher, K. B. Chandran, M. R. Crain, and Y. H. Kim, Am. J. Neuroradiol. 14, 66 (1993).

[15] K. Peldschus, M. Handorf, P. Robert, M. Port, G. Adam, and C. U. Herborn, J. Magn. Reson. Imaging 32, 459 (2010).

[16] A. W. Winfried, Eur. J. Radiol. 65, 2 (2008).

[17] M. Goyen, T. C. Lauenstein, C. U. Herborn, J. F. Debatin, S. Bosk, and S. G. Ruehm, J. Magn. Reson. Imaging 14, 602 (2001)

[18] S. Haneder, U. Attenberger, S. O. Schoenberg, C. Loewe, J. Arnaiz, H. J. Michaely, D. E. Mannheim, A. T. Vienna, and E. S. Santander, Eur. Congress Radiol. C1016 (2011). 